\title{
Developed Algorithm for Increasing the Efficiency of Data Exchange in a Computer Network
}

\author{
John S. N. \\ Covenant University \\ Department of Electrical and \\ Information Engineering, Ota, \\ Nigeria
}

\author{
Anoprienko A. A. \\ Donetsk State Technical \\ University, Artyom str., 58, \\ Donetsk, 83000, Ukraine
}

\author{
Okonigene R. E. \\ Ambrose Alli University, \\ Department of Electrical and \\ Electronics Engineering, \\ Ekpoma, Nigeria
}

\begin{abstract}
This paper presents specialized means to analyze, model and research of data exchange in large-scale corporate computer network. Due to extreme complexity of corporate intranet networks and the internet has resulted in the apparent difficulties in the development of an analytical model. Thus, under these circumstances, simulation models became viable alternative to comprehend the behavior of these complex networks during data exchange. This research work examined the mode of data exchange since its perfection allows in many cases to obtain a considerable improvement of the network and also the network application performance without substantial additional expenditure. Hence, the need for this developed algorithm for increasing the efficiency of data exchange in a computer network and the appropriate topology that suite this case. Test results from the algorithm showed an average of 10 to $15 \%$ increase and occasionally $60 \%$ and above increase in data exchange efficiency without additional expenses.
\end{abstract}

\section{General Terms}

Developed Algorithm

\section{Keywords}

Computer Network, Data Exchange, Bandwidth, Simulation, Efficiency

\section{INTRODUCTION}

The main task in analyzing and modeling of the modes of data exchange in modern computer networks based on the protocols of $\mathrm{TCP} / \mathrm{IP}$ is increasing the performance efficiency of work on the network and network application, and also increasing their productivity. Studies have shown that mode of data exchange contributed to the performance of a network by revealing the characteristic dependences of bandwidth capacity of a network from the work load of a corporate network based on the stack of the TCP/IP protocols $[1,2]$. Increasing the efficiency of data exchange in the computer networks based on the TCP/IP protocol requires difficult decision associate with different problems in which we have: choice and optimization of the network topology structure, optimization of the bandwidth capacity of communication channels, choice of routers, choice of methods in control management of data streams and determination of the management parameters, analysis of the buffer memory in commutation and router with choice of strategy in spooling during workloads [3, 4]. The study of computer network based on TCP/IP protocol requires determining the most effective topological structure within the framework that would result in the burst performance of the modes of exchange of information. The decisions touching on the topology of a network determine the basic descriptions of the network [5]. It is important to describe vividly the structure of a distributed computer network. Where an approximated result was required numerical method of analysis was recommended. Thus, the NetCracker Professional ${ }^{\circledR}$ environment was effective for low detailed analysis of computer networks based on standard network technology [6]. However, for non- standard protocols and devices and also for the necessity of detailed analysis, the use of Matlab/Simulink $®$ became handy [7, $8,9]$. This developed algorithm took advantage of the lapses of the discussed methods of analyses to improve the efficiency of computer network. This paper, therefore, described the method of how to increase the efficiency of data exchange in a network based on the developed algorithms.

\section{METHODOLOGY}

In order to increase the efficiency of data exchange in computer networks based on TCP/IP protocol required a detailed study of the: topological structure of the network, carrying capacity of communication channels, choice of routers, choice of methods of management by data streams and preset control parameters, analysis of the communication nodes, routers, and the choice of strategy of spooling at the overloads. After a review of various modes of exchange of data information in computer networks at different levels, simulated designs and analyses of these systems based on Matlab/Simulink and NetCracker Professional environments were executed. Results from different numerical analytical methods were obtained. Also included are the jointly developed corresponding different methods in designing and analyzing the network infrastructure with the main aim of providing an increase in the efficiency of data exchange based on the TCP/IP protocols stack.

\section{ANALYSIS OF THE OBTAINED RESULTS}

Figure 1 shows the summary of part of our research results carried out towards determining the rate of data loss during data exchange in a local area network (LAN) and the bandwidth efficiency of the network. In this case, a desired bandwidth was propagated toward the data flow and real bandwidth was obtained from the data streamline of the experiment. It was obvious, that in zone A the dependence of bandwidth on workload $Q_{T N}$ had a variable close to ideal network. Zone $\mathrm{B}$ is the zone of declination in network carriage capacity. Zone $\mathrm{C}$ is the saturation zone while zone $\mathrm{D}$ is the refusal area. It was observed that the mode of data exchange affects the mode in which computer networks operate, and also the effectiveness of the real bandwidth which was the throughput of the network. 


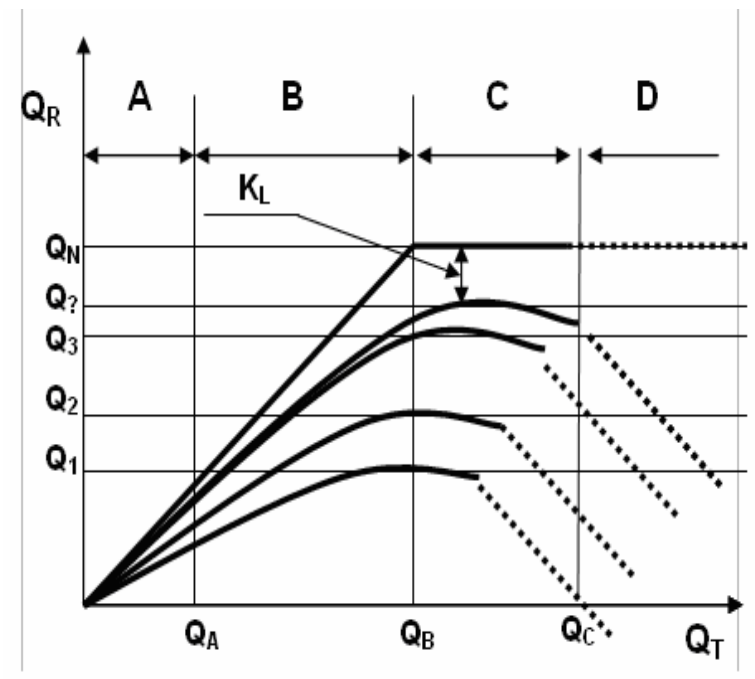

Figure 1 The effective usage of Computer Network

The generalized result obtained from Figure 1 has four zones of Network performance, as shown in Figure 2, which are:

$$
\begin{array}{lll}
\text { A } & \mathrm{Q}_{\mathrm{T}}<<\mathrm{Q}_{\mathrm{N}}, \text { hence } \mathrm{Q}_{\mathrm{R}}=\mathrm{Q}_{\mathrm{T},} & 1 \\
\mathrm{~B} & \mathrm{Q}_{\mathrm{T}}<\mathrm{Q}_{\mathrm{N}}, \text { hence } \mathrm{Q}_{\mathrm{R}}<\mathrm{Q}_{\mathrm{T}}, & 2 \\
\mathrm{C} & \mathrm{Q}_{\mathrm{T}} \geq \mathrm{Q}_{\mathrm{N}}, \text { hence } \mathrm{Q}_{\mathrm{R}}<\mathrm{Q}_{\mathrm{T}}, & 3 \\
\mathrm{D} & \mathrm{Q}_{\mathrm{T}} \gg \mathrm{Q}_{\mathrm{N}}, \text { hence } \mathrm{Q}_{\mathrm{R}}=0, & 4
\end{array}
$$

At $\mathrm{Q}_{\mathrm{T}} \approx \mathrm{Q}_{\mathrm{N}}$ the threshold of the network has $\mathrm{Q}_{\mathrm{R}}=\mathrm{Q}_{\text {Threshold}}$.

$\mathrm{Q}_{\mathrm{T}}$ - desired bandwidth, Mbps

$\mathrm{Q}_{\mathrm{N}}$ - channel bandwidth, Mbps

$\mathrm{Q}_{\mathrm{R}}$ - real bandwidth, Mbps

$\mathrm{Q}_{\mathrm{TN}}$ - workload

In zone A the network was observed to operate far below its bandwidth capacity. The real rate of data exchange was below the rate that it was predetermined to generate and the efficiency of the distributed environment was limited by the bandwidth of the network. The results therefore showed a substantial under utilization of computing possibilities of the distributed environment. Zones B and C are of upmost interest due to the fact that an actual bandwidth capacity substantially differs from the theoretical bandwidth because of the losses, caused by the working zones that are close to threshold point. Many factors are associated with the increase in size of packet losses that make their exact determination by theoretical methods impossible.

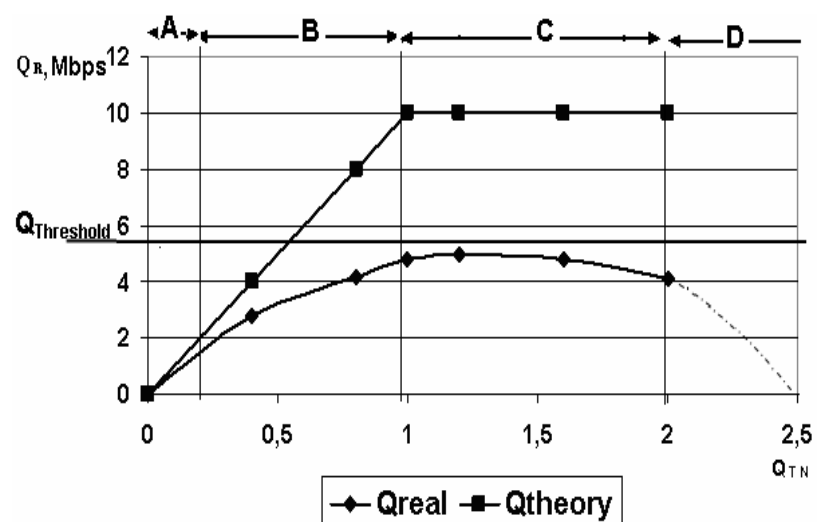

Figure 2 Dependence bandwidth of the network on the workload of a TCP/IP LAN
Hence, the use of simulated design method was considered to be appropriate. The $\mathrm{D}$ zone is characterized by complete degradation of the network.

Zones $\mathrm{B}$ and $\mathrm{C}$ represents the intensive changes of the coefficient of packet lost that can be determined with the following dependences:

$$
\mathrm{K}_{\mathrm{L}}=\mathrm{K}_{\mathrm{LS}}+\mathrm{K}_{\mathrm{LTCP}}
$$

Where, $\mathrm{K}_{\mathrm{L}}$ - coefficient of packet lost the header,

$\mathrm{K}_{\mathrm{LS}}-$ coefficient of packet lost from an exchange within

$\mathrm{K}_{\mathrm{LTCP}}$ - coefficient of packet lost from the features of $\mathrm{TCP} / \mathrm{IP}$ stack in the modes of network saturation.

The $\mathrm{K}_{\mathrm{LS}}$ is determined as follows:

$\mathrm{K}_{\mathrm{LS}}=\mathrm{N}_{\mathrm{K}} \cdot \mathrm{L}_{\mathrm{S}} / \mathrm{Q}_{\mathrm{T}}$ where, $N_{K}$ - the number of frames sent through the network in unit time:

$$
\mathrm{N}_{\mathrm{K}}=\mathrm{Q}_{\mathrm{R}} / \mathrm{L}_{\mathrm{F}}
$$

$\mathrm{L}_{\mathrm{S}}$ - number of headers in a frame in byte $\mathrm{L}_{\mathrm{F}}$ - size of data field in the frame $(50,100,500,1500$ bytes).

Thus, the evaluation of $\mathrm{K}_{\mathrm{LS}}$ was paramount and this was achieved by a model. In the case of small frame sizes (e.g. 100 byte) the $\mathrm{K}_{\mathrm{LS}}$ in $\mathrm{K}_{\mathrm{L}}$ was very substantial. But as the frame size was increased, the lost in the network performance was embedded in the $\mathrm{K}_{\mathrm{LTCP}}$ coefficient.

Theoretically, setting the $\mathrm{K}_{\mathrm{LTCP}}$ dependence on workload, mode of data exchange and other factors in a general case was impossible. Therefore, the basic method to evaluate the total lost in efficiency and the general efficiency of a network was the use of simulated models to obtain the proper dependences.

In the analysis of Figure 3 the dependence of the coefficient of packet lost from $\mathrm{Q}_{\mathrm{TN}}$ with different file sizes showed that increasing the workload increased the lost in efficiency in saturated mode $\left(\mathrm{Q}_{\mathrm{TN}}=0.8\right.$ to 1.6$)$. Consequently, therefore, there was an obvious lost in the overall efficiency of the network $\left(\mathrm{K}_{\mathrm{L}}=\right.$ 0.4 to 0.84 depending on the file size). The resultant lost in small file sizes as compared to larger file sizes was greater and this was due to the fact that more headers were added to each of the files during data exchange.

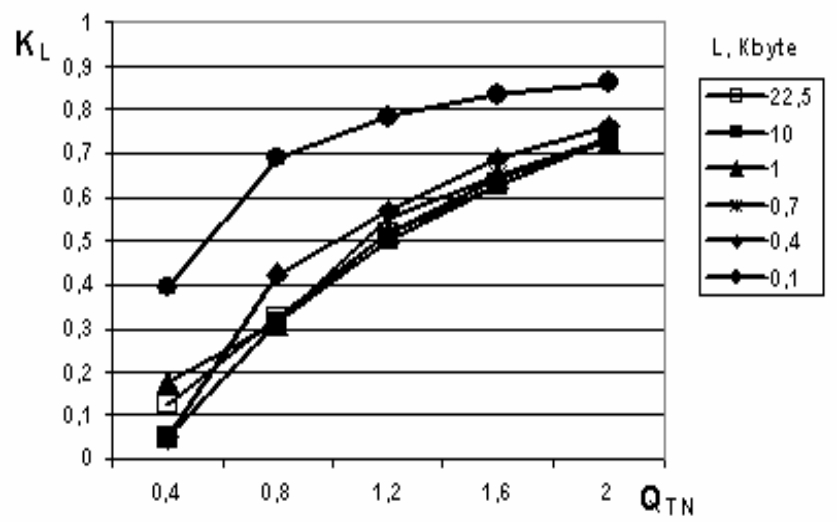

Figure 3 Dependence of $\mathrm{K}_{\mathrm{L}}$ on $\mathrm{Q}_{\mathrm{TN}}$ in the LAN

In our study of a corporate computer network in NetCracker Professional ${ }^{\circledR}$ environment the results showed that substantial losses in bandwidth efficiency depended on the passed file sizes and the amount of network nodes simultaneously involved in data transmission as shown in Figure 4. 


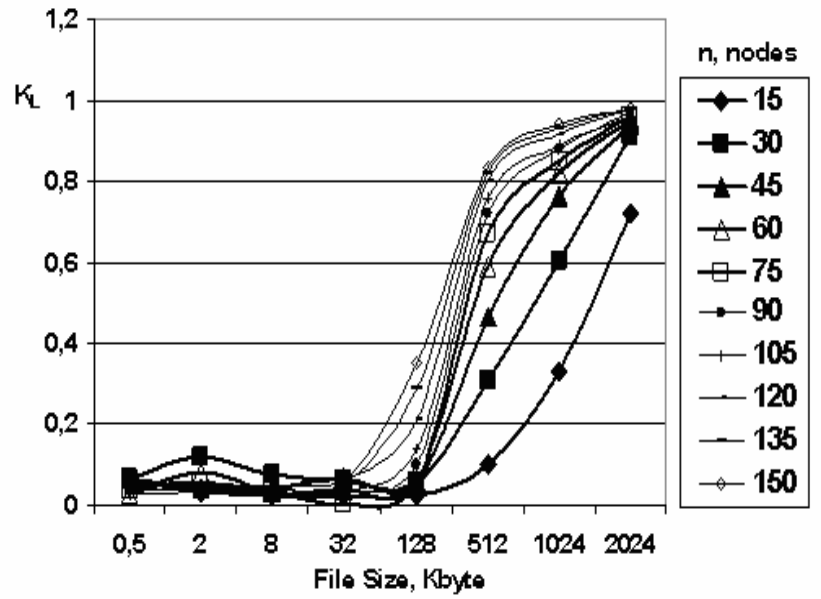

Figure 4 Dependence of $\mathrm{K}_{\mathrm{L}}$ on average passed file sizes

From the analyses of the results it was possible to obtain the threedimensional dependences represented in Figures 5, 6 and 7 for the mode of data exchange based on the bandwidth.

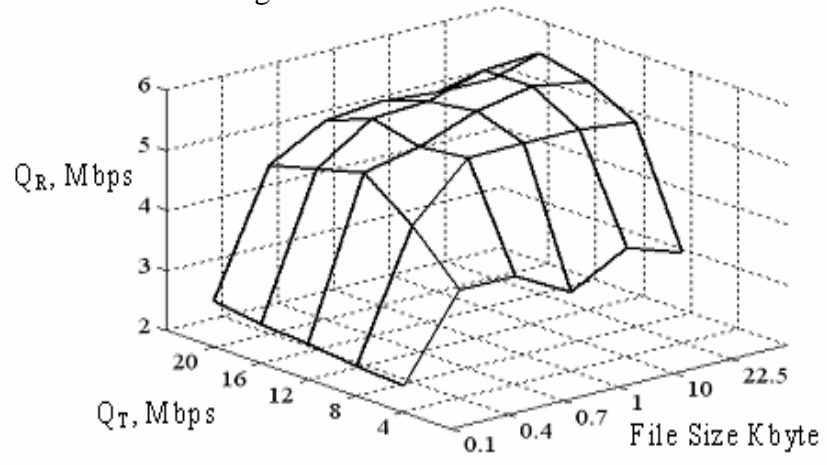

Figure 5 Dependence of Bandwidth in TCP/IP network on

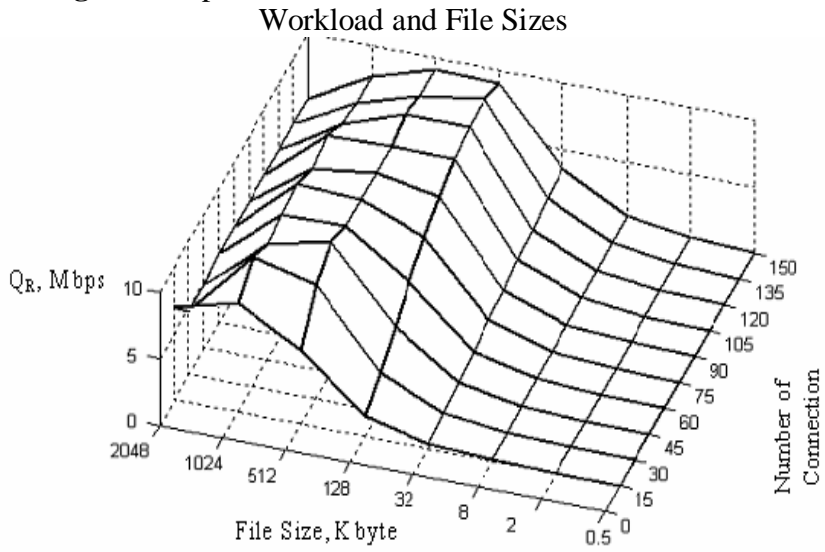

Figure 6 Dependence of Bandwidth on the number of network nodes and File Size

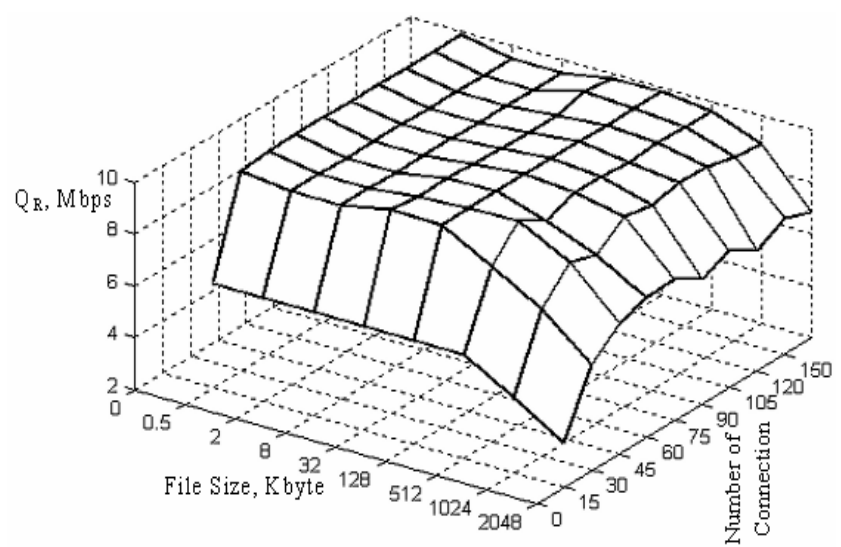

Figure 7 Dependence of Bandwidth on the number of network nodes and File Size at different time domain

Hence, the observed dependences in every case allowed the determination of the degree of influence of the modes of data exchange on the efficiency of the network. From our results it was apparent that simulated design gave details of how mode of data exchange can influence the efficiency of the network and how it can be used as basic element in developing the methods for increasing the efficiency of data exchange.

\section{THE DEVELOPED ALGORITHM}

Method of increasing the effectiveness of network data exchange based on using the simulated models is shown in Figure 8 and all the basic data, simulated models, the dependences with the analytical estimation and some crucial recommendations.

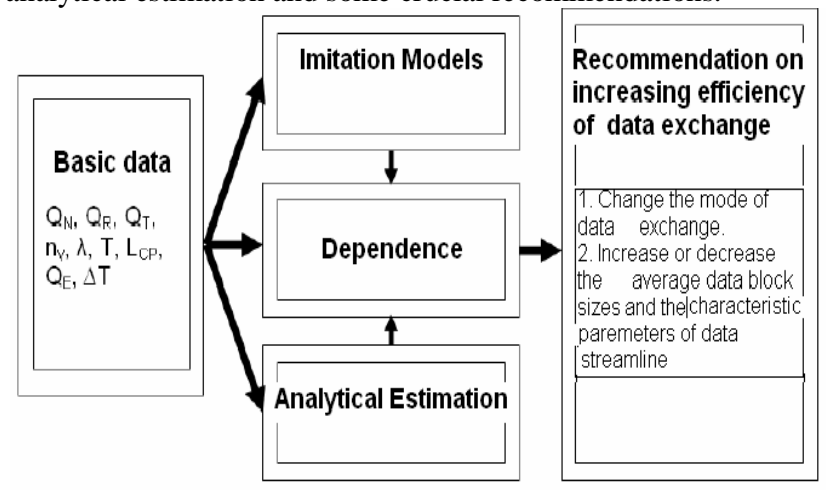

Figure 8 Method of increasing the effectiveness of network data exchange

The essence of the offered method consists in sharing the dependences obtained from analytical and simulated method, in the determination of the most efficient mode of data exchange.

1. The desired bandwidth of the network is:

$$
Q_{T}=\frac{I}{T}, \text { Kbyte } / \mathrm{sec}
$$

2. The Minimum rational file size is:

$$
L_{\text {min }}^{\text {Rat }}=\left[0.0507 \mathrm{Q}_{\mathrm{T}}-0.7833\right], \text { Kbyte }
$$

3. The maximal rational file size from (Fig. 6) is:

$L_{\max }^{\text {Rat }}=512 \frac{Q_{N}}{Q_{10}}$, Kbyte, 
where $\mathrm{Q}_{10}$ is data rate in the Ethernet network (1280 Kbyte/sec). 4. The Minimum rational period of data exchange is:

$$
\tau_{\min }^{\text {Rat }}=\frac{L_{\min }^{R a t} \cdot n_{c}}{Q_{T}},
$$

where $n_{c}=n_{y}$ is the number of network connections

5. The Maximal recommended period of data exchange is:

$$
\tau_{\max }^{\text {Rat }}=\frac{L_{\max }^{R a t} \cdot n_{c}}{Q_{T}}, \mathrm{sec} .
$$

where, $\mathrm{Q}_{\mathrm{E}}$ - effective bandwidth, $\mathrm{L}_{\mathrm{CP}}$ - average file size, $\mathrm{T}$ - time of data exchange, $\Delta \mathrm{T}$ - time delay at data exchange due to collisions and other problems, $\lambda$ - data frequency

\section{CONCLUSIONS}

The developed model was a fast method to determine the exact dependences in a computer network that are required to determine the efficiency parameters of data exchange to predetermine the corrections that are necessary to increase the efficiency of the network. We observed that analytical analysis gave relatively poor results and may be employed during the preliminary stage of the study. If standard technologies are employed it was recommended to use for low detailed analyses the NetCracker environment while for detailed analyses Matlab/Simulink. Our developed algorithm therefore was used to study computer networks modes of data exchange (data sizes passed, descriptions of block data streams), which resulted to an average of 10 to $15 \%$ and occasionally $60 \%$ and above increase in data exchange efficiency without additional expenses. In other words, a method was offered for increasing the efficiency of data exchange, which allows on the basis of the developed models and obtained dependences results to produce concrete suggestions on correction of the modes of data exchange with the purpose of increasing the efficiency functioning of the network. The specified dependences showed how in different modes of data exchange with growth of the desired bandwidth $\mathrm{Q}_{\mathrm{T}}$ changes the real bandwidth of the computer network $Q_{R}$. The $Q_{R}$ obtained dependence resulted from statistical analysis of the bandwidth of the TCP/IP network which has an exponential character.

\section{REFERENCES}

[1] S. N. John. 2005. Increasing the Efficiency of Data Exchange in a Computer Network Based on the Protocol of TCP/IP Suite, Scientific Journals: Information, cybernetics and Computing Engineering, Donetsk ( DonNTU), Ukraine, Vol. 93, pp. 256-264.

[2] John S. N., Anoprienko A. Y., Niru A. 2002. Multilevel simulation of networks on the base of TCP/IP protocols stack using Matlab/Simulink environment, Matlab/Simulink // Information cybernetic and computing texnika: Publ. 39, Scientific journal, Donestk: DonNTU, pp. 271-29.

[3] John S. N., Anoprienko A. Y., Rishka S. V. 2001. Simulating of university network infrastructure, Publ. KremeshuK State Technical University, Scientific journal KSTU, No. 2, vol. 11, Kremenshuk: KSTU, pp. 271-297.

[4] Olifer V. G., Olifer N. A. 1999. Computer network Principles of technologies, protocols, SPB: Publication «Inter».

[5] Vishnevski V. M. 2003. Theoretical bases of planning in computer networks, Tekhnosfera, Moscow, pp. $392-412$.

[6] Ponomarenko L. A., Shelkunov V.I., Sklrov A.A. 2002. Instrumental tools of planning, simulation design and analysis of computer networks. Kiev: Science Dumka,.

[7] Deaconov V. 2002. Simulink 4: Special reference book. Moscow.

[8] Guliteav A. K. 1999. Simulation design in Windows environment: Practical manual, SPB, KORONA.

[9] Simulink ${ }^{\mathrm{TM}}$. Accessed December 2009. Design and simulate continuous- and discrete-time systems available at http://www.mathworks.com/products/Simulink ${ }^{\mathrm{TM}}$. 